\title{
Optimal Modes for the Fabrication of Aluminum Nanopowders by the Electrical Explosion of Wires
}

\author{
Alexei Pustovalov, ${ }^{1}$ Vladimir An, ${ }^{1}$ and Jin-Chun Kim ${ }^{2}$ \\ ${ }^{1}$ Institute of High Technology Physics, National Research Tomsk Polytechnic University, 30 Lenin Ave., Tomsk 634050, Russia \\ ${ }^{2}$ School of Materials Science and Engineering, University of Ulsan, 93 Daehak-ro, Nam-Ku, Ulsan 44610, Republic of Korea \\ Correspondence should be addressed to Vladimir An; an_vladimir@tpu.ru
}

Received 26 July 2016; Revised 12 December 2016; Accepted 11 January 2017; Published 8 February 2017

Academic Editor: Antonio Riveiro

Copyright (C) 2017 Alexei Pustovalov et al. This is an open access article distributed under the Creative Commons Attribution License, which permits unrestricted use, distribution, and reproduction in any medium, provided the original work is properly cited.

\begin{abstract}
The paper is aimed at studying the impact of initial conditions of electrical explosion of wires on energy characteristics of the explosion and some other properties of the obtained aluminum powders. Explosion modes where the energy input into the wire has the maximal level were found. These modes are optimal for fabrication of powders with the best properties. The powders have the highest value of the specific surface of $14.5 \mathrm{~m}^{2} / \mathrm{g}$, a narrow histogram of the particle size distribution, and a narrow distribution histogram with a high polydispersity coefficient of 0.7 .
\end{abstract}

\section{Introduction}

Metal nanopowders are used in the development of highquality construction materials. Because of their structural peculiarities, the products of powder metallurgy are more thermally stable and resist better cyclic temperature gradients and deformation stress [1]. The transition from bulk metals to highly dispersed powders and nanoparticles is characterized by a change in a number of fundamental material properties, such as melting temperature, electron work function, and chemical reactivity [2]. This allows us to prepare novel metal and composite materials with improved mechanical, electrophysical, magnetic, and physicochemical characteristics. In addition, these nanopowders are used as highly active catalysts $[3,4]$, lubricants $[5,6]$, and activators of combustion and sintering $[7,8]$.

One of the fabrication methods for metal nanopowders is the electrical explosion of wires (EEW) [8]. EEW is characterized by low energy consumption related to the pulsed supply of energy to the wire, technology simplicity, and the availability of raw materials. EEW is carried out in an inert gas and experiment parameters are empirically found in order to produce pure metal nanopowders. So far, the level of the energy input into the wire during EEW has been seen to exercise the most significant influence over the obtained powder dispersivity [9]. However, a great number of variables such as the capacity of capacitor battery, the initial voltage of its charge, the diameter and length of the exploded wire, circuit inductance, and gas pressure complicate the calculation of EEW in ensuring the desired mode of explosion for fabrication of powders with chosen wires.

The objective of the present work is to study the influence of the energy input into the wire and the initial parameters of the electrical explosion of aluminum wires on the properties of produced aluminum nanopowders.

\section{Methods and Approach}

Experiments on electrical explosion of aluminum wires were carried out in the setup described in [10]. To simplify the experiments, EEW was performed at constant capacity of $1.08 \mu \mathrm{F}$, circuit inductance of $0.82 \mu \mathrm{H}$, and argon pressure of $3 \cdot 10^{5} \mathrm{~Pa}$. Aluminum wire of $0.25 \mathrm{~mm}$ in diameter was used for the experiments.

The starting voltage was varied from 20 to $30 \mathrm{kV}$, and the exploded wire length was varied from 30 to $180 \mathrm{~mm}$. The response on a change of parameters was controlled with waveforms recorded using a current shunt and an oscilloscope Tektronix 2014B. Additionally, the EEW current and voltage waveforms were treated in order to calculate the 


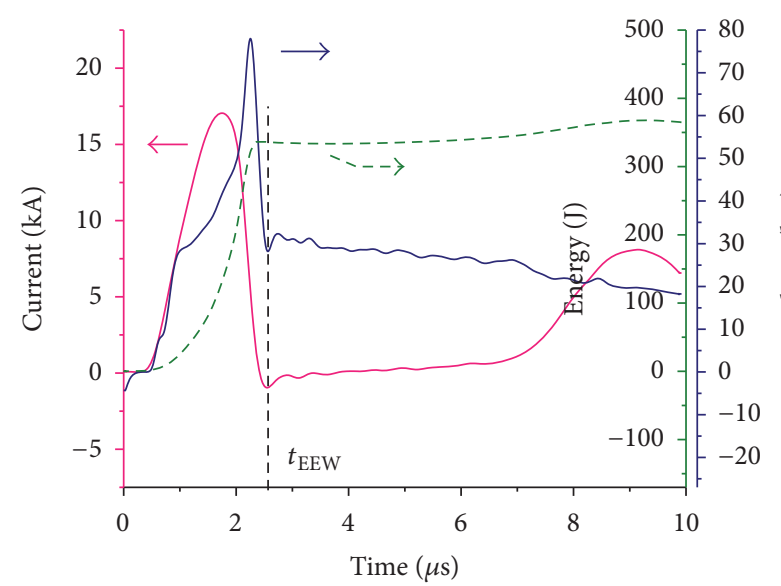

(a)

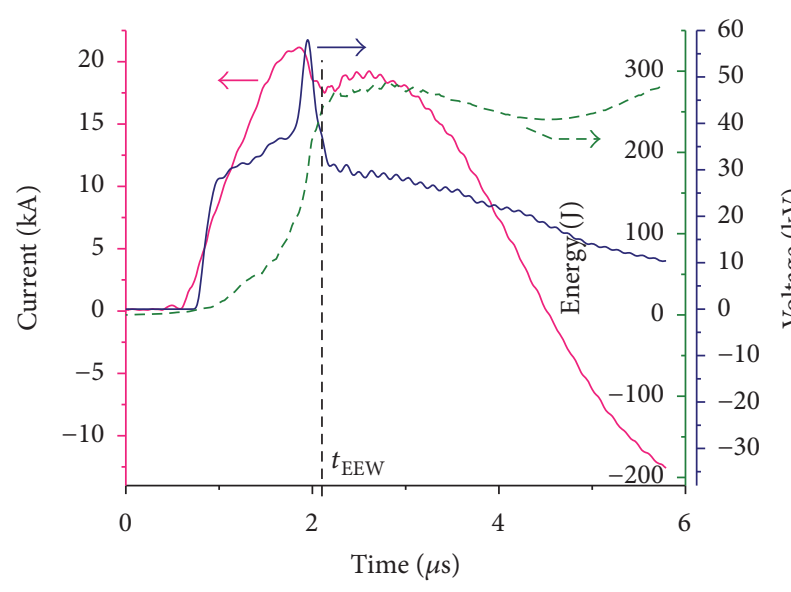

(c)

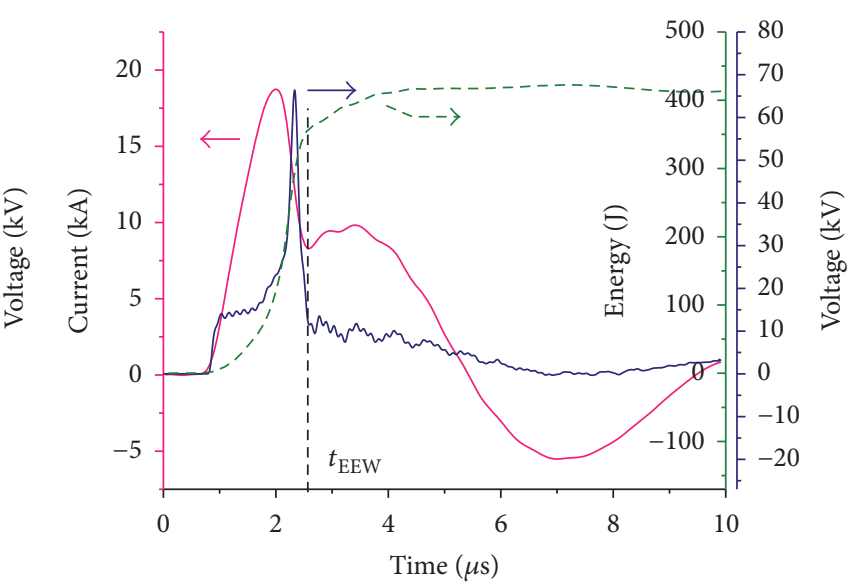

(b)

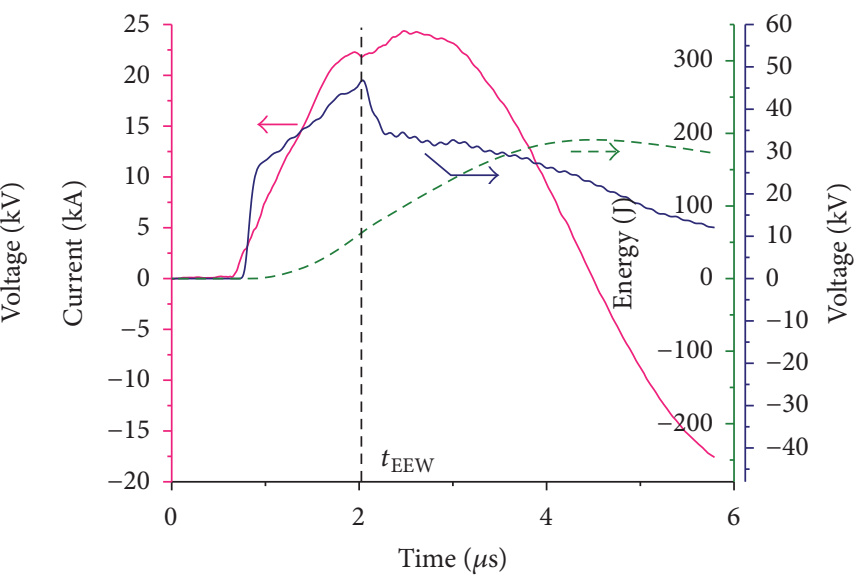

(d)

FIGURE 1: The EEW current and voltage waveforms: $U_{0}=30 \mathrm{kV}$. (a) $l=175 \mathrm{~mm}, e / e_{S}=1.1$, and $e_{\text {arc }} / e_{S}=0.2 ;$ (b) $l=135 \mathrm{~mm}, e / e_{S}=1.6$, and $e_{\text {arc }} / e_{S}=0.4$; (c) $l=85 \mathrm{~mm}, e / e_{S}=2.0$, and $e_{\text {arc }} / e_{S}=1.2 ;$ (d) $l=60 \mathrm{~mm}, e / e_{S}=1.7$, and $e_{\text {arc }} / e_{S}=2.8$.

energy input into the wire and also the energy released during the charge arc stage. The energy released in the discharge gap in the range of time from the beginning of the current rise until the inflection moment of the current waveform $\left(t_{\mathrm{EEW}}\right.$ in Figure 1) was considered as the energy input into the wire and the rest of the released energy as that released during the arc discharge stage.

The energy input into the wire was calculated using (1) offered by the authors of [11]:

$$
\begin{aligned}
W_{\mathrm{EEW}}= & \frac{C U_{0}^{2}}{2}-\frac{C\left(U_{0}-(1 / C) \int_{0}^{t_{\mathrm{EEW}}} i(t) \mathrm{d} t\right)^{2}}{2} \\
& -\frac{L \cdot i\left(t_{\mathrm{EEW}}\right)^{2}}{2}-R \int_{0}^{t_{\mathrm{EEW}}} i(t)^{2} \mathrm{~d} t,
\end{aligned}
$$

where $C$ is the capacitor capacity, $U_{0}$ is the voltage of capacitor charge, $i$ is the EEW current, $L$ is the inductance of setup circuit, $R$ is the circuit resistance, and $t_{\mathrm{EEW}}$ is the duration of an EEW current pulse. According to the authors of [11], the accuracy of the calculations of the energy input into the wire is not less than $4-6 \%$. The calculations did not take into account the inductance of the exploded wire which was not less than $10 \%$. In order to compare the various modes of electrical explosion of wires, the energy input into the wire was expressed in the form of a dimensionless value:

$$
\frac{e}{e_{s}}=\frac{W_{\mathrm{EEW}}}{V_{W} \cdot e_{S}},
$$

where $e$ is the specific energy, $V_{W}$ is the volume of the exploded wire, and $e_{S}$ is the energy of aluminum sublimation $\left(32.9 \mathrm{~J} / \mathrm{mm}^{3}\right)$. The energy released during the charge arc stage was also converted into a dimensionless value $e_{\mathrm{arc}} / e_{s}$.

The obtained aluminum powders were passivated in air using the method described in [12]. The shape and the average size of the particles were studied using a transmission electron microscopy JEM-2100 F (JEOL, Japan). A particle size distribution histogram was additionally built. For this purpose, more than 15 microphotographs obtained at different magnitudes (totally more than 3000 particles) were treated. This histogram served for the calculations of the number-average $\left(a_{n}\right)$, surface-average $\left(a_{S}\right)$, and mass-average $\left(a_{m}\right)$ particle size. According to $a_{n} / a_{m}$ ratio, the particle 
polydispersion coefficient $(k)$ characterizing the particle size "dispersion" was calculated.

The specific surface area $\left(S_{\mathrm{sp}}\right)$ was measured according to the BET method using a Sorbtometer-M device. The specific surface area was used for the calculations of the surface-average particle diameter according to the following equation:

$$
d_{S}=\frac{6}{\rho S_{\mathrm{sp}}} \cdot 1000[\mathrm{~nm}],
$$

where $\rho$ is the density of aluminum, $2.7 \mathrm{~g} / \mathrm{cm}^{3}$.

The phase composition was determined using an X-ray diffractometer, Shimadzu XRD-7000 (Japan). According to the data of X-ray peak maximum enlargement, the size of coherent scattering regions (average size of crystallites) was calculated using the Debye-Scherrer equation:

$$
d_{\mathrm{CSR}}=\frac{k \cdot \lambda}{\beta \cdot \cos (2 \theta / 2)},
$$

where $k$ is the dimensionless coefficient (for spherical particles it is 0,9$), \lambda$ is the $\mathrm{X}$-ray wavelength $(0.1540593 \mathrm{~nm}), \beta$ is the full width at half maximum, and $2 \theta$ is the diffraction angle.

The content of metallic aluminum $\left(\mathrm{Al}^{0}\right)$ in samples was measured using the volumetric method during interaction of the metal with a $10 \% \mathrm{NaOH}$ solution.

\section{Results and Discussion}

Figure 1 shows the EEW current and voltage waveforms. This figure also illustrates the dependence of the energy input during the discharge period versus time at a fixed voltage of $30 \mathrm{kV}$. In this experiment, the only exploded wire length was varied from 175 to $30 \mathrm{~mm}$.

Variation in the exploded wire length leads to a rather high change in the EEW waveforms. At the longest length (Figure 1(a)), the EEW mode is characterized by two separately occurring processes. The first process is electrical explosion itself. The second one is the EEW arc stage. This explosion mode is characterized by a high coefficient of energy transfer from the capacitor to the wire: more than $80 \%$. In the literature, this mode is called mode with a «current pause» $[9,13]$. Nevertheless, the value of the energy input into the wire is not high and is equal to $330 \mathrm{~J}\left(1.1 e_{S}\right)$. The decrease of the exploded wire length up to $85 \mathrm{~mm}$ leads to fusion of electrical explosion itself and the discharge arc stage: a transition to the mode without current pause $[9,13,14]$. The level of the energy input into the wire decreases down to $270 \mathrm{~J}$. However, an increase in the specific energy (up to $2 e_{S}$ ) into the wire $2 e S$ is observed due to an increase in the exploded wire volume. Moreover, the energy released during the arc discharge stage increases up to $1.2 e_{S}$ (Figure 1(c)). Then, when decreasing the length (Figure 1(d)), a bigger part of energy is released during the arc stage, whereas the specific energy input into the wire decreases down to $1.7 e_{S}(130 \mathrm{~J})$. Probably, it is due to a rather low electrical strength of argon. When the wire length is less than $80 \mathrm{~mm}$, an earlier start of the arc

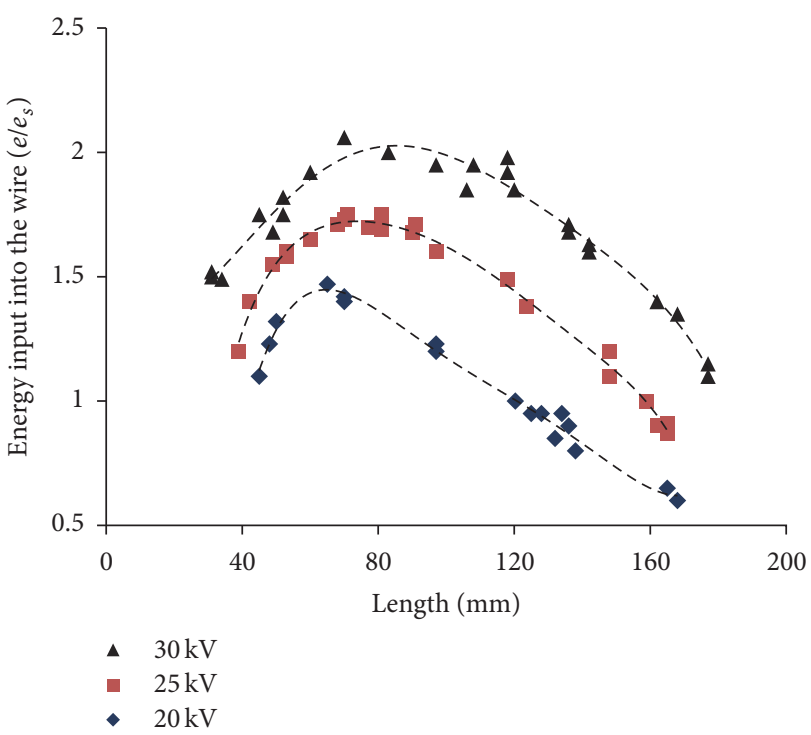

FIGURE 2: Energy input into the wire during explosion versus the exploded wire length and the voltage.

discharge stage is observed. It leads to shunting of the energy input into the wire (Figure 1(d)).

Figure 2 depicts the dependence of the specific energy input into the wire during the explosion on the exploded wire length for a series of experiments carried out at a voltage of 20,25 , and $30 \mathrm{kV}$.

The regularities of the change of $e / e_{S}$ from $l$ at different $U_{0}$ reveal similar character. The minimal $e / e_{S}$ value is observed during electrical explosion of wires with the maximal length. A decrease in the exploded wire length leads to an increase in $e / e_{S}$. However, an increase in the specific energy input into the wire is observed up to a definite value of the working voltage. For the series of experiments at $U=20 \mathrm{kV}$, the maximal $e / e_{S}$ value (1.5) is observed at $l=65 \mathrm{~mm}$. For the series of experiments at $U=30 \mathrm{kV}$, the maximal $e / e_{S}$ value is 2 at $l \approx 80 \mathrm{~mm}$. The further increase of the wire length leads to a fast decrease of the energy input into the wire.

Initial EEW conditions for fabrication of nanopowders were chosen taking into account the obtained data. The initial EEW conditions and properties of the powders produced under these conditions are given in Table 1.

Sample Al-1, obtained at the lowest level of the specific energy input into the wire during the explosion $\left(0.9 e_{S}\right)$, has the lowest specific surface area that equals $5.6 \mathrm{~m}^{2} / \mathrm{g}$. The particles have a spherical shape; there are both big particles of $4.5 \mu \mathrm{m}$ and small particles of 25 to $400 \mathrm{~nm}$. According to the literature data [15], the big particles form during the metal melting and sputtering, whereas the origin of the small particles is related to the metal evaporation during the explosion and the further condensation of the vapors. On the particles surface, we observe an approximately $5 \mathrm{~nm}$ thick well-defined layer which consists of aluminum oxidehydroxide phases according to the literature data [2] formed during the passivation of the powders in the air. The presence of these phases is confirmed by the volumetric analysis, 

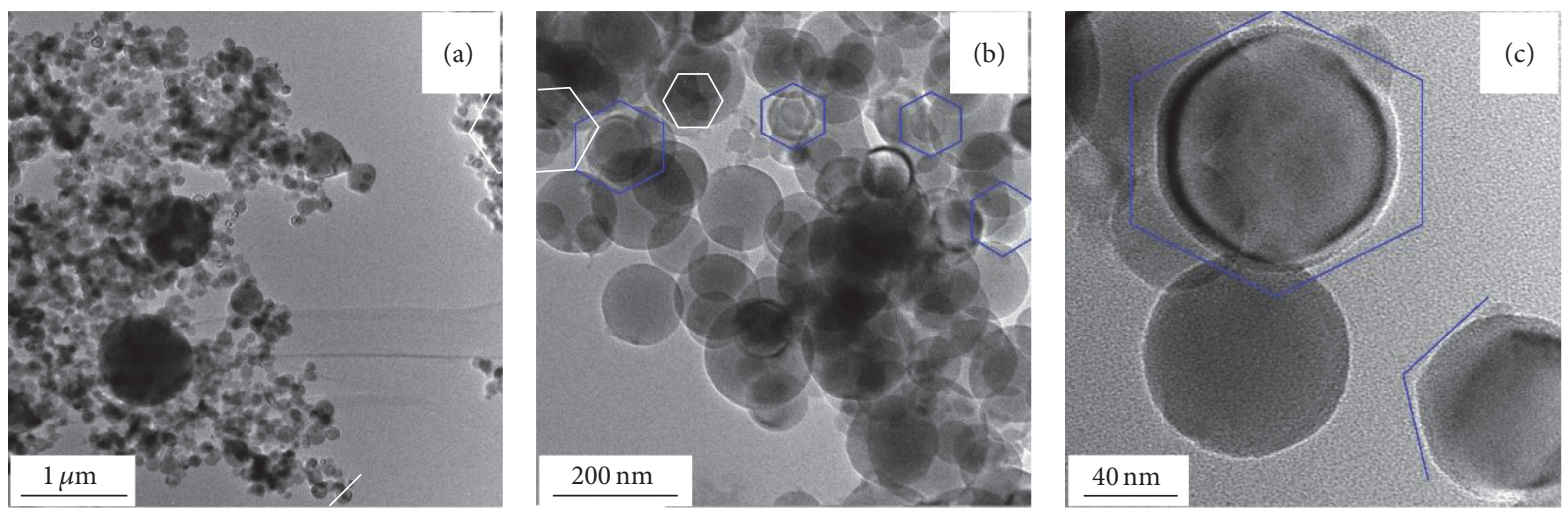

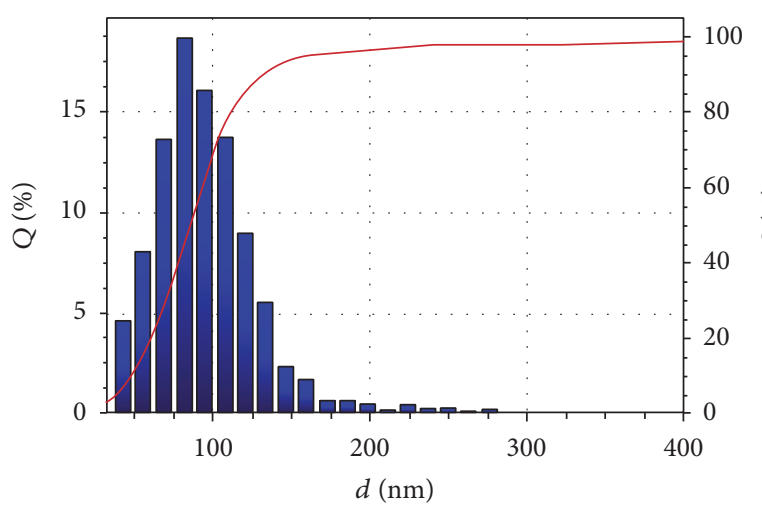

(d)

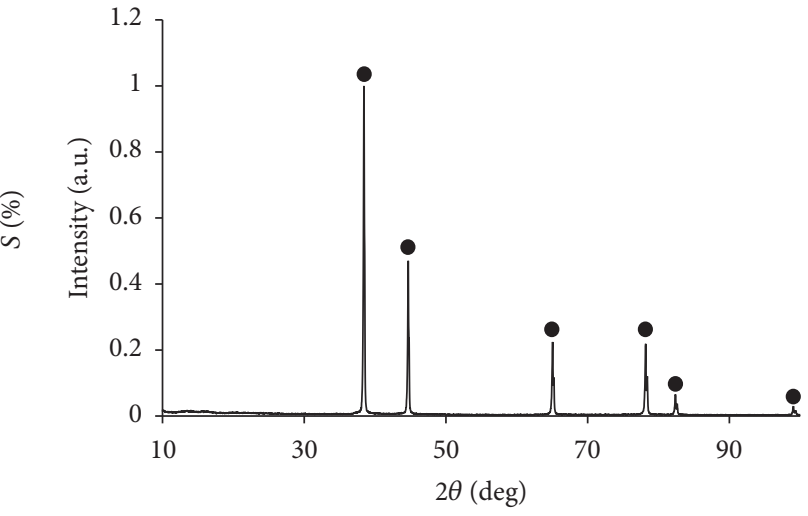

Al-04-0787

FIGURE 3: Microphotographs (a, b, c), histogram of the particle size distribution (d), and XRD pattern (e): sample Al-5: $a_{n}=130 \mathrm{~nm}$; $a_{S}=$ $150 \mathrm{~nm} ; a_{m}=180 \mathrm{~nm} ; k=0.7$.

TABLE 1: Impact of EEW energetic parameters on the characteristics of Al powders.

\begin{tabular}{|c|c|c|c|c|c|c|c|}
\hline Sample & $U_{0}, \mathrm{kV}$ & $l, \mathrm{~mm}$ & $e / e_{S}$ & $e_{\mathrm{ars}} / e_{S}$ & $S_{\mathrm{sp}}, \mathrm{m}^{2} / \mathrm{g}$ & $d_{s}, \mathrm{~nm}$ & $\mathrm{Al}^{0}$, wt. $\%$ \\
\hline Al-1 & 20 & 120 & 0.9 & 0.1 & 5.6 & 400 & 96 \\
\hline $\mathrm{Al}-2$ & 20 & 75 & 1.4 & 0.2 & 8.1 & 275 & 93 \\
\hline $\mathrm{Al}-3$ & 25 & 120 & 1.5 & 0.1 & 9.2 & 240 & 92 \\
\hline $\mathrm{Al}-4$ & 25 & 75 & 1.7 & 0.8 & 11.2 & 200 & 91 \\
\hline Al-5 & 30 & 85 & 2.0 & 1.2 & 14.5 & 150 & 89 \\
\hline Al-6 & 30 & 60 & 1.7 & 2.8 & 13.9 & 160 & 90 \\
\hline $\mathrm{Al}-7$ & 30 & 30 & 1.5 & 7.5 & 13.1 & 170 & 90 \\
\hline
\end{tabular}

according to which the content of metallic aluminum in the sample is $96 \mathrm{wt} . \%$.

The size of $95 \%$ of the particles ranges from 25 to $400 \mathrm{~nm}$; their maximum distribution corresponds to $115 \mathrm{~nm}$. The presence of $5 \%$ of particles with a size from $400 \mathrm{~nm}$ to $4.5 \mu \mathrm{m}$ leads to an increase in the number of particles of average size up to $160 \mathrm{~nm}$ and up to $350 \mathrm{~nm}$ in the weight-average.

The sample is characterized by a rather low polydispersity coefficient: 0.4 . When the particle size distribution is large, the surface-average particle diameter, calculated from the distribution histogram $\left(a_{S}\right)$, is practically 2 times lower than the analogous value calculated from the specific surface area $\left(d_{S}\right)$. The difference between $a_{S}$ and $d_{S}$ can be explained by the difference in techniques of sample preparation for analysis. The BET measurements are more accurate because the surface of all particles is measured in contrast of TEM, where most of big particles, sedimented in the solution, are not analyzed.

The increase in $e / e_{S}$ leads to an increase in the specific surface area of the obtained particles. Highest $S_{\text {sp }}$ was found for the samples prepared under the modes with the maximal level $e / e_{S}$ equal to 2.0 (Al-5, Table 1). The microphotographs of sample Al-5 are given in Figure 3. The sample is characterized by a narrow range of the particle size distribution and a rather high polydispersity coefficient: 0.7 . As a result, the surfaceaverage particle diameter, calculated from the distribution histogram, corresponds to the particle size calculated using the powder specific surface area. The size of most particles is 
in the range of $25-200 \mathrm{~nm}$, whereas particles of approx. $1 \mu \mathrm{m}$ are observed but their part is not high.

Most particles of nanometer size have a spherical shape. However, in contrast to the previous sample, we can observe particles having the shape of a hexagon (Figures 3(b) and $3(\mathrm{c})$ ), where the diameter of such particles is always less than $100 \mathrm{~nm}$. According to the authors [2], the presence of the faceted particles (white hexagonal or polygonal lines in Figures 3(b) and 3(c)) is related to the mechanism of their crystallization. Droplets with the size of 100 or more nanometers crystallize from a large number of nuclei of which the mutual growth limitations contribute to the formation of particles with a spherical shape. Crystallization of relatively small droplets can occur during the formation and growth of one or a few nuclei when the particle forms in the form of a hexagon.

The increase in the specific surface area of the studied samples from $5.6 \mathrm{~m}^{2} / \mathrm{g}$ to $14.5 \mathrm{~m}^{2} / \mathrm{g}$ leads to a twofold decrease in the sizes of coherent scattering regions from $150 \mathrm{~nm}$ to $70 \mathrm{~nm}$ which can be explained by a decrease in crystallite sizes. However, the crystallite size for the studied samples calculated using the Debye-Scherrer formula is lower than the average particle size calculated using the powder specific surface area (Table 1). This allows us to assume that the powder particle produced at $2.0 e_{S}$ has a polycrystalline structure.

The study of the modes with an increase of $e / e_{S}$ and fast rise of $e_{\mathrm{arc}} / e_{S}$ showed that the powder specific surface area under these explosion modes decreases with a decrease in $e / e_{S}$ (samples Al-6 and Al-7, Table 1). The powder particles obtained at the highest energy released during the arc discharge stage (sample Al-7) have both a spherical shape and polygon shape. The difference between this sample and the one considered above consists in the appearance of particles sintered with each other. Probably it is due to the release of large amount of discharge arc stage heat resulting in the sintering of particles.

\section{Conclusions}

An increase in the specific energy input into the wire is observed when decreasing the length of the exploded aluminum wire and keeping constant the other EEW conditions. However, this increase occurs until a definite moment. The dependence of $e / e_{S}$ on $U_{0}$ and $l$ has a maximum; the further decrease in the length leads to either significant increase in the working voltage or increase in the energy released at the discharge arc stage and decrease in the specific energy input into the wire during the explosion.

The optimal EEW modes for the fabrication of metal Al powders in the studied range are the explosion modes with the maximal level of the energy input into the wire $(U=$ $30 \mathrm{kV} ; l=85 \mathrm{~mm}$ ). The powders have the highest value of the specific surface of $14.5 \mathrm{~m}^{2} / \mathrm{g}$, a narrow histogram of the particle size distribution, and a high polydispersity coefficient.

Under the modes with an increase in the specific energy input into the wire during the arc discharge stage, the specific surface area decreases; the samples were found to have a high amount of sintered particles which can be provoked by the high thermal action of the energy released during the discharge arc stage.

\section{Competing Interests}

The authors declare that there are no competing interests regarding the publication of this paper.

\section{Acknowledgments}

This study was supported by the Russian Foundation for Basic Research (Grant RFBR-mol-a 16-38-00051). This work was also supported under the state assignment of the Ministry of Education and Science of Russia for 2014-2016 (Research Work no. 361). The authors would like to thank the NanoCenter and Scientific Analytical Centre at Tomsk Polytechnic University for the XRD, TEM, and SEM analyses. It is their pleasure to express their thanks to Ms. Mary Page for her invaluable help in correcting the manuscript.

\section{References}

[1] R. E. Smallman and A. H. W. Ngan, Physical Metallurgy and Advanced Materials, Butterworth-Heinemann, 2011.

[2] A. V. Korshunov, "Influence of dispersion aluminum powders on the regularities of their interaction with nitrogen," Russian Journal of Physical Chemistry A, vol. 85, no. 7, pp. 1202-1210, 2011.

[3] P. Rauwel, S. Küünal, S. Ferdov, and E. Rauwel, "A review on the green synthesis of silver nanoparticles and their morphologies studied via TEM," Advances in Materials Science and Engineering, vol. 2015, Article ID 682749, 9 pages, 2015.

[4] S. L. Suib, Ed., New and Future Developments in Catalysis: Batteries, Hydrogen Storage and Fuel Cells, Newnes, 2013.

[5] V. An, Y. Irtegov, and C. De Izarra, "Study of tribological properties of nanolamellar WS $\mathrm{W}_{2}$ and $\mathrm{MoS}_{2}$ as additives to lubricants," Journal of Nanomaterials, vol. 2014, Article ID 865839, 8 pages, 2014.

[6] V. An and Y. Irtegov, "Tribological properties of nanolamellar $\mathrm{MoS}_{2}$ doped with copper nanoparticles," Journal of Nanomaterials, vol. 2014, Article ID 731073, 7 pages, 2014.

[7] G. V. Sakovich, V. A. Arkhipov, A. B. Vorozhtsov, S. S. Bondarchuk, and B. V. Pevchenko, "Investigation of combustion of HEM with aluminum nanopowders," Nanotechnologies in Russia, vol. 5, no. 1, pp. 91-107, 2010.

[8] Y.-S. Kwon, Y.-H. Jung, N. A. Yavorovsky, A. P. Illyn, and J.S. Kim, "Ultra-fine powder by wire explosion method," Scripta Materialia, vol. 44, no. 8-9, pp. 2247-2251, 2001.

[9] Y. A. Kotov, "The electrical explosion of wire: a method for the synthesis of weakly aggregated nanopowders," Nanotechnologies in Russia, vol. 4, no. 7-8, pp. 415-424, 2009.

[10] A. V. Pustovalov, "Study of products of electrical explosion of iron wires in argon-oxygen mixture," Advanced Materials Research, vol. 872, pp. 206-213, 2014.

[11] I. F. Kvartskhava, V. V. Bondarenko, A. A. Pliutto, and A. A. Chernov, "Oscillographic determination of energy of electric explosion of wires," Journal of Experimental and Theoretical Physics, vol. 31, pp. 745-775, 1956.

[12] A. A. Gromov, U. Förter-Barth, and U. Teipel, "Aluminum nanopowders produced by electrical explosion of wires and passivated by non-inert coatings: characterisation and reactivity with 
air and water," Powder Technology, vol. 164, no. 2, pp. 111-115, 2006.

[13] C. S. Wong, B. Bora, S. L. Yap, Y. S. Lee, H. Bhuyan, and M. Favre, "Effect of ambient gas species on the formation of $\mathrm{Cu}$ nanoparticles in wire explosion process," Current Applied Physics, vol. 12, no. 5, pp. 1345-1348, 2012.

[14] Y. S. Lee, B. Bora, S. L. Yap, and C. S. Wong, "Effect of ambient air pressure on synthesis of copper and copper oxide nanoparticles by wire explosion process," Current Applied Physics, vol. 12, no. 1, pp. 199-203, 2012.

[15] Y. Kinemuchi, K. Murai, C. Sangurai et al., "Nanosize powders of aluminum nitride synthesized by pulsed wire discharge," Journal of the American Ceramic Society, vol. 86, no. 3, pp. 420424, 2003. 

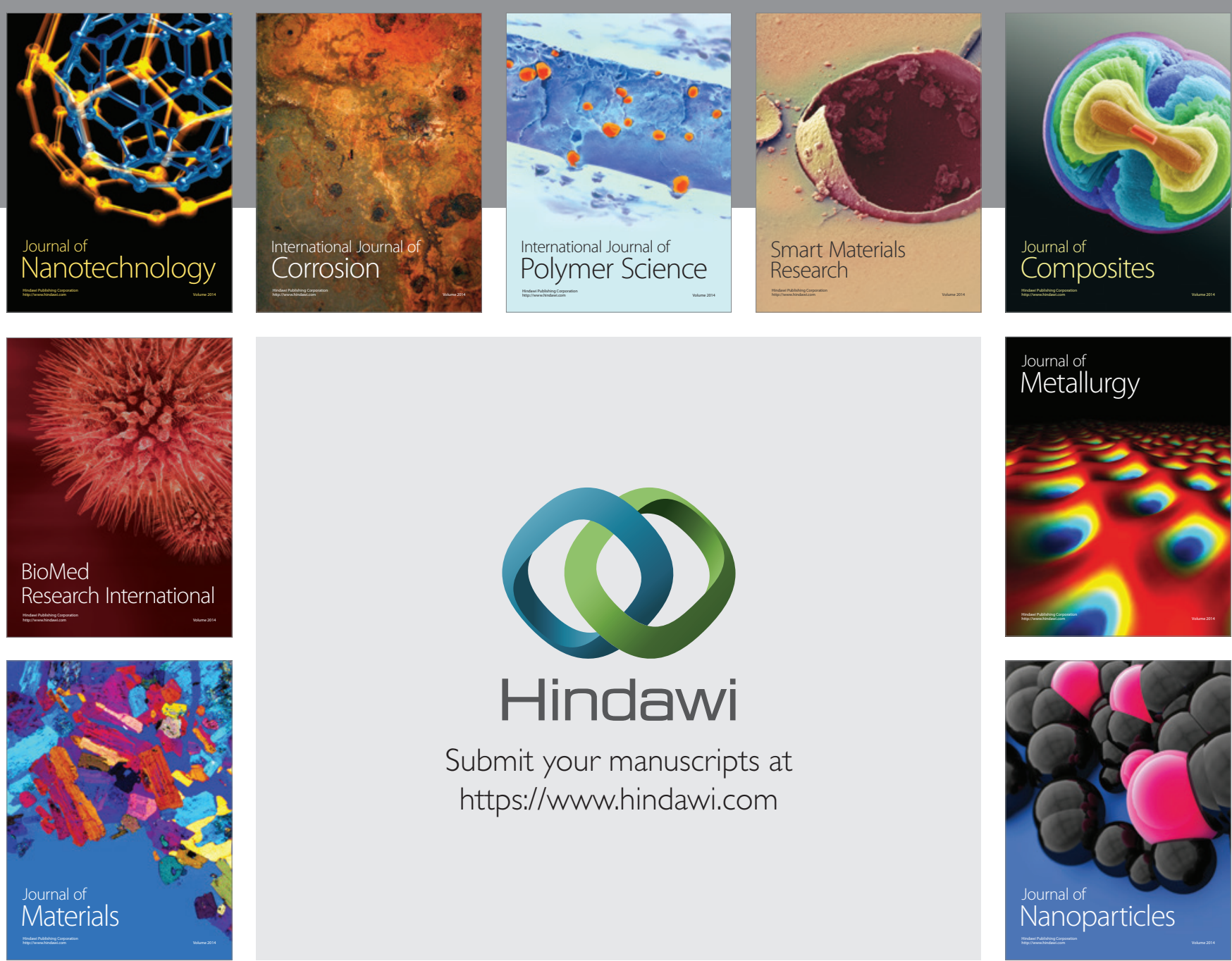

\section{Hindawi}

Submit your manuscripts at

https://www.hindawi.com

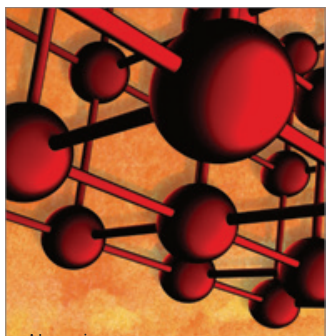

Materials Science and Engineering
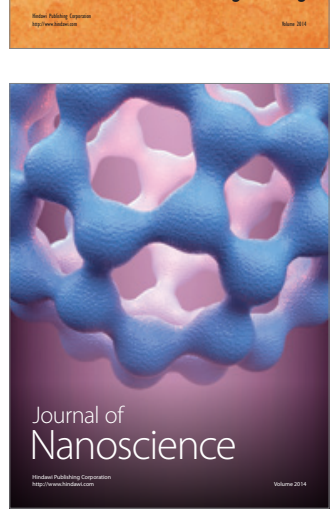
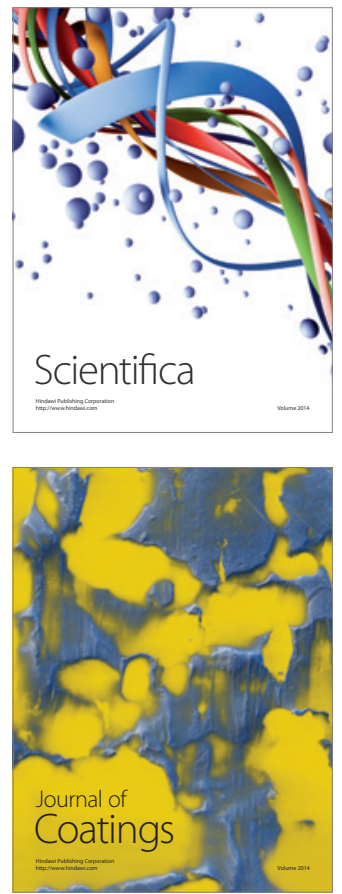
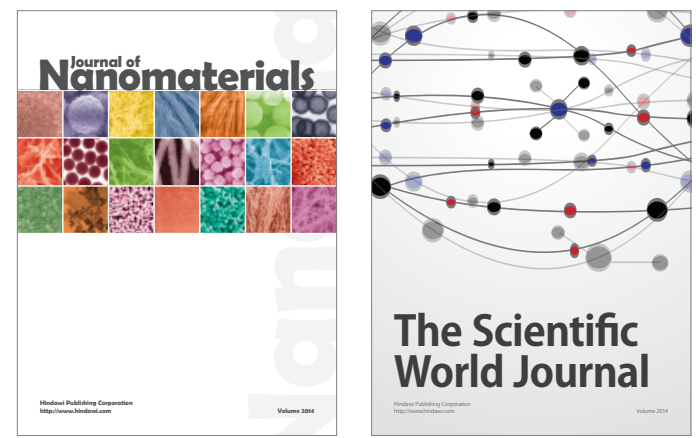

The Scientific World Journal
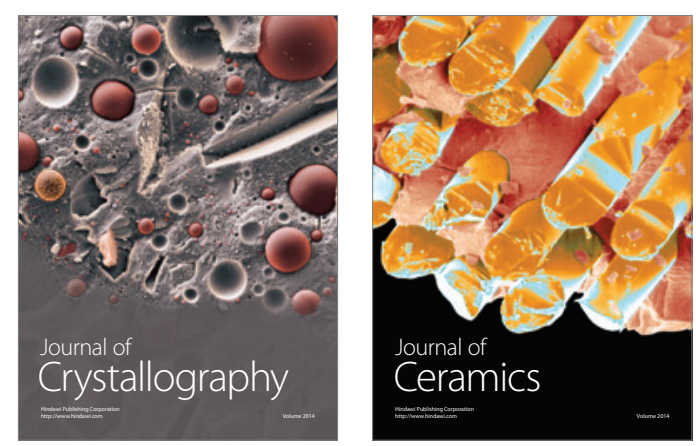
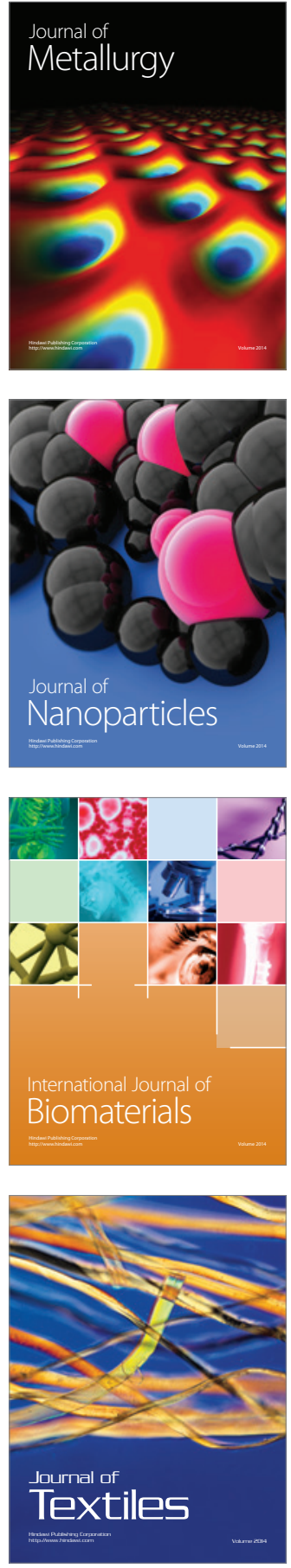